\title{
Las fuentes del crecimiento económico mexicano, 1988-2004
}

DOI: $10.32870 /$ mycp.v8i26.263

Germán Alarco Tosoni ${ }^{1}$

Introducción

$\mathrm{P}$ ara evaluar la efectividad de la política económica de cualquier país o territorio, es importante conocer cómo las fuentes del crecimiento económico inciden en el desempeño macroeconómico. El objetivo principal de este trabajo es presentar y estimar varias metodologías que resaltan los factores que explican el crecimiento económico de México durante 1988-2004. ${ }^{1}$ Aplicamos diferentes metodologías empíricas para analizar los resultados económicos de las tres últimas administraciones: Salinas de Gortari, Zedillo y Fox. La idea es contrastar sus propuestas de política económica con los resultados observados y los sucesos que no pudieron controlar.

Como las metodologías usadas en este trabajo se basan en la oferta agregada, la demanda agregada y los ingresos no son plenamente compatibles entre sí. Diferentes factores influyen en el crecimiento del producto interno bruto (PIB): la dinámica de los sectores productivos — según su clasificación convencional-; los choques de ingresos como el incremento de los precios del petróleo y las remesas de los emigrantes mexicanos o la apreciación del tipo de cambio, la cual eleva el poder de

* Profesor de la Universidad Panamericana, México, DF. El autor agradece los valiosos comentarios de Patricia del Hierro Carrillo y el apoyo de Rafael Hernández Parra en el trabajo estadístico. Email: germanalarco@yahoo.com.mx. compra de los residentes nacionales bajo una economía abierta; la expansión de la demanda interna, externa y la sustitución de importaciones; la política monetaria y fiscal; entre otros.

Cabe aclarar que ninguna de las metodologías sugeridas está sujeta a una comprobación estadística estricta que permita rechazar o aceptar las hipótesis respectivas. Tampoco se hace un análisis histórico exhaustivo del desempeño de las distintas administraciones gubernamentales ni los resultados de éstas se confrontan con las políticas llevadas a cabo. Además, el análisis de las fuentes del crecimiento omite los elementos inerciales que son importantes durante los primeros años de cada administración y de igual manera excluye la estimación de funciones de producción por divergir con la teoría subyacente. ${ }^{2}$

La estructura del trabajo es la siguiente. La primera sección muestra el crecimiento del PIB total per cápita y por periodos. También evalúa la contribución de la oferta y la demanda al crecimiento del producto. La segunda utiliza una clasificación diferente basada en el sector agropecuario y de hidrocarburos, la industria maquiladora de exportación, la construcción y el resto de actividades productivas. La tercera sección explica el crecimiento en función de la demanda interna y externa, y la sustitución de importaciones. La cuarta aborda la influencia de algunos choques externos como los incrementos de los precios de los 
hidrocarburos y las remesas. La quinta sección enfatiza el papel de las políticas fiscal y monetaria: se trata de un ejercicio experimental en sí. La última sección discute y cuantifica la influencia de la sobrevaluación del peso en el crecimiento económico.

\section{Los resultados generales y el análisis tradicional según la oferta y la demanda}

La información estadística oficial muestra claramente que las tasas de crecimiento del PIB y del PIB per cápita han sido positivas pero reducidas, y que su tendencia ha sido decreciente desde el gobierno de Salinas de Gortari; en lo que va de la administración de Fox la tasa de crecimiento per cápita es ligeramente superior a cero. Sin embargo, si tomamos una perspectiva más amplia apreciamos que ha habido avances en cuanto al ingreso-producto promedio real de los últimos 16 años.

La contribución al crecimiento de cada actividad productiva $\left(S_{i}\right)$ se obtiene con la diferencia de su aportación entre los años considerados, $T$ y $T-\alpha$, respecto a la variación absoluta del PIB real (base $=1993)$ para cada periodo gubernamental y del total 1988-2004 $\left(P_{I B_{T}}-P I B_{T-\alpha}\right)$. La agregación de las aportaciones sectoriales individuales es igual a $100 \%$ :
(1) $\sum_{i}^{n}\left[\frac{\left(S_{i, T}-S_{i, T-\alpha}\right)}{\left(P I B_{T}-P I B_{T-\alpha}\right)} * 100\right]=100$

En este caso hemos trabajado con las clásicas nueve divisiones del producto que se consolidan en tres grandes grupos: sector primario (agropecuario, silvicultura, pesca y minería), secundario (industria manufacturera, construcción, electricidad y agua) y terciario (comercio, restaurantes, hoteles, transporte, almacenamiento, comunicaciones, servicios financieros, seguros, bienes inmuebles, servicios comunales, sociales y personales). Una última cuenta de ajuste agrupa a los impuestos con los productos netos y los servicios bancarios imputados.

En todo el periodo destaca la contribución del sector terciario con $67.2 \%$ del total, seguido del secundario con $24.9 \%$ y del primario con $4.4 \%$ (tabla 2). Dentro del terciario resalta la participación del comercio, restaurantes y hoteles con $20.4 \%$, los servicios financieros, seguros y bienes inmuebles con $18.8 \%$ y transporte, almacenamiento y comunicaciones con $17.1 \%$. En el caso del sector secundario destaca la industria manufacturera con $19.8 \%$, la construcción con $3.4 \%$ y electricidad, gas y agua con $1.8 \%$. El sector agropecuario, silvicultura y pesca explica sólo $3.6 \%$ del crecimiento económico, mientras que la minería $0.8 \%$.

Tabla 1

Las tasas de crecimiento del PIB agregado y per cápita, 1988-2004 (En porcentajes)

\begin{tabular}{lcccc}
\hline & Salinas, & Zedillo, & Fox, & Total, \\
& $1988-1994$ & $1994-2000$ & $2000-2004$ & $1988-2004$ \\
\hline Crecimiento acumulado del PIB & 25.87 & 22.37 & 6.52 & 64.08 \\
piba & 3.91 & 3.42 & 1.59 & 3.14 \\
pib per cápita & 11.45 & 10.94 & 0.05 & 23.70 \\
pib per cápitab & 1.82 & 1.75 & 0.01 & 1.34 \\
\hline
\end{tabular}

a. Tasa de crecimiento promedio anual.

b. Tasa de crecimiento acumulado

Fuente: elaboración propia con base en Fox (2005). 
Si bien durante los periodos de Salinas de Gortari y Zedillo la producción fue impulsada por la participación más equilibrada de los tres sectores, durante la administración de Fox el sector terciario explica casi todo el crecimiento del PIB con $96.3 \%$, mientras que la participación del primario fue de $10 \%$ y del secundario de $-1.7 \%$. En los últimos cuatro años los sectores financiero (bancos y seguros) y de bienes inmuebles explican $40.6 \%$ del bajo crecimiento, seguidos del transporte, almacenamiento y comunicaciones con $34.4 \%$, y del comercio, restaurantes y hoteles con $16.4 \%$. La industria manufacturera contribuyó con $-6.5 \%$, que fue contrarrestado parcialmente por la industria de la construcción con $2.9 \%$ y de la electricidad, gas y agua con $1.9 \%$.

Durante todo el periodo destaca la mayor contribución del consumo privado seguido de las exportaciones, la formación bruta de capital privado y público y el consumo del gobierno. Las mayores contribuciones negativas (filtraciones de la demanda) son las importaciones de bienes y servicios con un mayor valor que el de las exportaciones y la variación de existencias. La estimación de estos resultados sustituye la información sectorial $\left(S_{i}\right)$ por la de los componentes de la demanda $\left(C D_{i}\right)$ en la ecuación (1):
(2) $\sum_{i}^{n}\left[\frac{\left(C D_{i, T}-C D_{i, T-\alpha}\right)}{\left(P I B_{T}-P I B_{T-\alpha}\right)} * 100\right]=100$

De acuerdo con la tabla 3, existen algunas diferencias sobresalientes. Durante el periodo de Salinas de Gortari destacan las contribuciones del consumo privado, las exportaciones, la formación bruta de capital privado y público, el consumo público y la variación de existencias. Después, en el periodo de Zedillo sobresale la expansión de las exportaciones debida al Tratado de Libre comercio de América del Norte (TLCAN) y al ajuste cambiario de 1994, en tanto que la aportación del consumo privado es menor y la de la formación bruta de capital público es negativa. En la administración de Fox prevalecen el consumo privado y la formación bruta de capital público, y, a su vez, la negativa participación de la formación bruta de capital privado y la variación de existencias. Salinas de Gortari logró movilizar la inversión privada, Zedillo lo hizo más o menos y Fox no ha podido.

Es común que todos los gobiernos pretendan atribuir sus problemas a los factores externos para mejorar el crecimiento económico. La tabla 4 reclasifica la información de la tabla 3 en factores internos de economía cerrada — consumo privado, consumo público, formación bruta

Tabla 2

La contribución de los sectores productivos al crecimiento, 1988-2004 (\%)

\begin{tabular}{|c|c|c|c|c|c|c|}
\hline & \multirow[t]{2}{*}{ TCPA (\%)a } & \multicolumn{3}{|l|}{ Sectores } & \multirow{2}{*}{$\begin{array}{c}\text { Impuestos a los productos } \\
\text { menos servicios bancarios } \\
\text { imputados }\end{array}$} & \multirow[t]{2}{*}{ Tota } \\
\hline & & Primario & Secundario & Terciario & & \\
\hline Salinas, 1988-1994 & 3.91 & 3.11 & 26.10 & 67.71 & 3.08 & 100 \\
\hline Zedillo, 1994-2000 & 3.42 & 3.60 & 33.40 & 56.44 & 6.55 & 100 \\
\hline Fox, 2000-2004 & 1.59 & 10.04 & -1.70 & 96.27 & -4.61 & 100 \\
\hline Total, 1988-2004 & 3.14 & 4.41 & 24.95 & 67.24 & 3.40 & 100 \\
\hline
\end{tabular}

a. TCPA $=$ tasa de crecimiento promedio anual.

Fuente: elaboración propia con datos de Fox (2005). 
Análisis

Tabla 3

La influencia de la demanda agregada en el crecimiento, 1988-2004

(En porcentajes)

\begin{tabular}{|c|c|c|c|c|c|c|c|c|}
\hline & \multirow[t]{2}{*}{$\begin{array}{l}\text { TCPA } \\
(\%)\end{array}$} & \multicolumn{2}{|c|}{ Consumo } & \multicolumn{2}{|c|}{$\begin{array}{l}\text { Formación bruta } \\
\text { de capital }\end{array}$} & \multirow{2}{*}{$\begin{array}{l}\text { Variación } \\
\text { de } \\
\text { existencias }\end{array}$} & \multicolumn{2}{|c|}{$\begin{array}{c}\text { Balanza comercial de } \\
\text { bienes y servicios }\end{array}$} \\
\hline & & Privado & Gobierno & Privada & Pública & & Exportación & Importación \\
\hline Salinas & 3.91 & 86.46 & 8.60 & 24.46 & 8.99 & 4.60 & 30.14 & -63.26 \\
\hline Zedillo & 3.42 & 55.17 & 5.10 & 31.64 & -3.82 & 3.02 & 115.35 & -106.46 \\
\hline Fox & 1.59 & 130.38 & -4.09 & -9.53 & 13.29 & -31.75 & 64.21 & -62.51 \\
\hline $\begin{array}{l}\text { Total, } \\
1988-2004\end{array}$ & 3.14 & 79.59 & 5.08 & 22.29 & 4.04 & -1.79 & 72.93 & -82.13 \\
\hline
\end{tabular}

Fuente: elaboración propia con datos de Fox (2005).

de capital privado y público, y variación de existencias - y factores externos - diferencia entre exportaciones e importaciones de bienes y servicios-. En este sentido, en el periodo 1988-2004 las fuentes del crecimiento son básicamente internas, en tanto que en las administraciones de Zedillo y Fox la contribución del sector externo fue positiva y en la de Salinas negativa.

Ahora clasificamos las fuentes de la demanda de acuerdo con la naturaleza del impulso: en endógenos - consumo privado, formación bruta de capital privado, variación de existencias e importaciones de bienes y servicios ${ }^{3}$ - y exógenos —inyecciones de demanda: consumo público, formación bruta de capital público y exportaciones de bienes y servicios-. Observamos que el crecimiento durante el periodo Zedillo se explica por factores exógenos, el de Salinas es principalmente endógeno y el de Fox se ubica en una situación intermedia.

\section{La contribución de acuerdo con algunas actividades seleccionadas}

A diferencia de lo presentado en el primer apartado sobre la contribución de los sectores productivos al crecimiento, ahora se incorpora la información de la industria maquiladora de exportación y de los hidrocarburos. Cabe aclarar que no restamos la participación de las importaciones de bienes intermedios de la maquila equivalente a $76.2 \%$ de su valor bruto de la producción (VBP), incluimos otras actividades econó-

Tabla 4

Los factores del crecimiento clasificados por la naturaleza de sus componentes, 1988-2004 (En porcentajes)

\begin{tabular}{lrrrrrrr}
\hline & \multicolumn{2}{c}{ Economía cerrada y abierta } & \multirow{2}{*}{ Total } & \multicolumn{2}{c}{ Factores endógenos y exógenos } & \multirow{2}{*}{ Total } \\
\cline { 2 - 3 } & Internos & Externos & & Endógenos & Exógenos & \\
\hline Salinas & 133.12 & -33.12 & 100 & 52.26 & 47.74 & 100 \\
Zedillo & 91.11 & 8.89 & 100 & -16.63 & 116.63 & 100 \\
Fox & 98.30 & 1.70 & 100 & 26.59 & 73.41 & 100 \\
Total, 1988-2004 & 109.20 & -9.20 & 100 & 17.96 & 82.04 & 100 \\
\hline
\end{tabular}

Fuente: elaboración propia con datos de Fox (2005). 
micas que no se traslapan con la maquila y obtenemos residualmente el resto de los sectores productivos. ${ }^{4}$

En todo el periodo la industria maquiladora de exportación - sin deducir sus importaciones de insumos-explica $40.6 \%$ del crecimiento económico. En el periodo de Zedillo fue equivalente a $65.1 \%$ de ese crecimiento, mientras que durante el de Salinas fue de $17 \%$. En una situación intermedia se ubica la administración Fox con $32.3 \%$ del crecimiento observado (tabla 5). Aunque la contribución del sector hidrocarburos es pequeña durante todo el periodo, ésta alcanza su mayor aportación durante la administración Fox con poco más de 1\%.

Llama la atención que el sector de la construcción haya logrado su mayor contribución durante la administración Salinas, seguida por las de Fox y Zedillo. Como se comentó, el sector agropecuario, en tanto una parte del primario, logró su mayor contribución al crecimiento durante la administración de Fox. En todo el periodo el resto de los sectores productivos explicó $51.6 \%$ de la expansión del PIB.

\section{La demanda interna y externa, y la penetración de las importaciones}

Con la metodología de Chenery dividimos las diferentes contribuciones al crecimien- to económico en demanda interna $(D)$, demanda externa $(B)$, importaciones complementarias asociadas al nivel del PIB y las sustitutas que corresponden a los cambios en la propensión a importar $(\mathrm{m})$ como resultado de las políticas comercial y cambiaria - una versión aproximada de esta metodología fue aplicada por Cárdenas (1994).

La ecuación (3) especifica las tres fuentes del crecimiento: demanda interna, demanda externa e importaciones. La demanda interna incluye el consumo privado, el consumo público, la formación bruta de capital privado, la formación bruta de capital público y la variación de existencias. La demanda externa comprende las exportaciones de bienes y servicios. La ecuación (4) indica que las importaciones son el resultado de la propensión marginal a importar en proporción del PIB. Posteriormente, la ecuación (5) incorpora la ecuación (4) en la $(3)$ y se expresa en variaciones $(\Delta)$. El tercer componente - ubicado en el lado derecho de la ecuación- se refiere a las importaciones complementarias y el cuarto y el quinto a las importaciones sustitutas. La ecuación (6) presenta la fórmula con variables discretas y distribuye entre sus diferentes componentes explicativos el crecimiento económico de todo el periodo analizado.

Tabla 5

La contribución al crecimiento de algunos sectores productivos, 1988-2004

(En porcentajes)

\begin{tabular}{|c|c|c|c|c|c|c|}
\hline & Agropecuario & Hidrocarburos & Maquila & Construcción & $\begin{array}{l}\text { Resto de } \\
\text { sectores }\end{array}$ & Total \\
\hline Salinas & 2.54 & 1.02 & 17.00 & 6.23 & 73.21 & 100 \\
\hline Zedillo & 2.76 & 0.70 & 65.14 & 0.96 & 30.44 & 100 \\
\hline Fox & 8.53 & 1.04 & 32.25 & 2.90 & 55.28 & 100 \\
\hline Total, 1988-2004 & 3.58 & 0.88 & 40.55 & 3.39 & 51.60 & 100 \\
\hline
\end{tabular}

Fuente: elaboración propia con datos de Fox (2005). 
(3) $P I B+M=D I+D E$

(4) $M=m P I B$

(5) $\triangle P I B=\triangle D I+\triangle D E-\triangle P I B m-$ $\triangle m P I B-\triangle m \Delta P I B$

(6) $\left[\left(\frac{D I_{T}-D I_{T-\alpha}}{P I B_{T}-P I B_{T-\alpha}}\right)+\left(\frac{D E_{T}-D E_{T-\alpha}}{P I B_{T}-P I B_{T-\alpha}}\right)-m_{T-\alpha}-\right.$ $\left.\left(\frac{\left(m_{T}-m_{T-\alpha}\right) * P I B_{T-\alpha}}{P I B_{T}-P I B_{T-\alpha}}\right)-\left(m_{T}-m_{T-\alpha}\right)\right] * 100=100$

La tabla 6 indica que durante el periodo analizado la demanda interna fue el principal factor que explicó el crecimiento seguido de la demanda externa. Las importaciones complementarias y las sustitutas contribuyeron negativamente. Como se dijo, la demanda externa se maximizó durante la administración de Zedillo, y las importaciones sustitutas, como resultado de la mayor propensión a importar, participaron negativamente durante el gobierno de Fox. Pero las autoridades podrían revertir tal tendencia, sobretodo con las importaciones de bienes de consumo y de capital. ${ }^{5}$

\section{Los flujos de ingresos provenientes de los hidrocarburos y las remesas de los emigrantes}

Esta sección pretende evaluar la contribución de las exportaciones no petroleras y de las petroleras al crecimiento económico, para lo cual distingue las variaciones en los volúmenes de crudo exportados y sus precios. Asimismo, busca evaluar la contribución de las remesas de los emigrantes mexicanos en el exterior. $\mathrm{Al}$ respecto, es importante señalar dos características de los hidrocarburos y las remesas: explican las variaciones del producto y están fuera del control de las autoridades mexicanas.

El cálculo de la contribución de las exportaciones o demanda externa $(D E)$ se hace con las exportaciones no petroleras $(E N P)$ y petroleras $(E P)$. El valor de las exportaciones petroleras se calcula multiplicando el precio de la mezcla mexicana de exportación $(P)$ por el volumen exportado $(V)$, como lo muestra la ecuación (7). Luego agregamos sus variaciones y los dividimos por la variación absoluta del producto - la suma de estos componentes debe ser igual a 100- La ecuación 9 desglosa todos los factores explicativos en el lado izquierdo: las exportaciones no petroleras, los precios del petróleo crudo y las variaciones de los volúmenes exportados.

(8)

$\frac{\Delta D E}{\Delta P I B}=\frac{\Delta E N P}{\Delta P I B}+\frac{\Delta P V}{\Delta P I B}+\frac{\Delta P \Delta V}{\Delta P I B}+\frac{\Delta V P}{\Delta P I B}$

(9)

$$
\left[\left(\frac{E N P_{T}-E N P_{T-\alpha}}{P I B_{T}-P I B_{T-\alpha}}\right)+\left(\frac{\left(P_{T}-P_{T-\alpha}\right) V_{t-\alpha}}{P I B_{T}-P I B_{T-\alpha}}\right)+\right.
$$

$\left.\left(\frac{\left(P_{T}-P_{T-\alpha}\right)\left(V_{T}-V_{T-\alpha}\right)}{P I B_{T}-P I B_{T-\alpha}}\right)+\left(\frac{\left(V_{T}-V_{T-\alpha}\right) P_{t-\alpha}}{P I B_{T}-P I B_{T-\alpha}}\right)\right] * 100=1$

La mayor contribución de las exportaciones de bienes y servicios al crecimiento económico se produjo durante el gobierno de Zedillo, seguido de la administración de Fox y por último la de Salinas (tabla 7). En la segunda columna de la tabla $7 \mathrm{se}$ aprecia que el crecimiento de las exportaciones de bienes y servicios no petroleras explicó la mayor expansión de las exportaciones, aunque fuera decreciente. En la administración de Zedillo explicó 94\% del crecimiento de las exportaciones, mientras que en el gobierno de Fox fue de $81.4 \%$ de la variación de las exportaciones. Las variaciones de los volúmenes exportados tienen 
Tabla 6

Las fuentes del crecimiento clasificadas por la demanda y las importaciones, 1988-2004 (En porcentajes)

\begin{tabular}{lccccc}
\hline & $\begin{array}{c}\text { Demanda } \\
\text { interna }\end{array}$ & $\begin{array}{c}\text { Demanda } \\
\text { externa }\end{array}$ & $\begin{array}{c}\text { Importaciones } \\
\text { complementarias }\end{array}$ & $\begin{array}{c}\text { Importaciones } \\
\text { sustitutas }\end{array}$ & Total \\
\hline Salinas & 133.12 & 30.14 & -51.60 & -11.66 & 100 \\
Zedillo & 91.11 & 115.35 & -84.20 & -22.27 & 100 \\
Fox & 98.30 & 64.21 & -24.85 & -37.66 & 100 \\
Total, 1988-2004 & 109.20 & 72.93 & -70.47 & -11.66 & 100 \\
\hline
\end{tabular}

Fuente: elaboración propia con datos de Fox (2005).

poca relevancia en el caso de los periodos de Salinas y Zedillo porque la plataforma de exportación permaneció prácticamente fija, mientras que en la administración de Fox explicaron $8.1 \%$ de las exportaciones. Las variaciones en los precios de la mezcla mexicana de petróleo crudo aportan, en lo que va de este gobierno, $10.6 \%$ del total de las variaciones en las exportaciones, equivalentes a $6.8 \%$ de la variación del producto interno bruto. En el gobierno de Salinas la aportación fue negativa, mientras que durante el de Zedillo fue equivalente a $5.1 \%$ de la variación del producto.

Las administraciones de Zedillo y Fox gozaron de coyunturas favorables en los precios internacionales del petróleo, los cuales elevaron los ingresos, la demanda y la producción. Sin embargo, los precios de los hidrocarburos son una variable exógena que no puede ser controlada por la política económica del país.

Para evaluar la contribución al crecimiento económico de las remesas de los emigrantes mexicanos en el exterior, debemos leer de manera diferente la cuenta corriente de la balanza de pagos: la diferencia entre las importaciones y exportaciones de bienes y servicios es equivalente al ahorro externo, y, conjuntamente con el ahorro privado y público interno, es la contrapartida de la formación bruta de capital público y privado. En este sentido, el desequilibrio de nuestra cuenta corriente representa la contribución de los individuos y empresas de otros países a nuestro ahorro, inversión ${ }^{6}$ y, por lo tanto, al crecimiento económico. Un mayor ahorro externo implica que los

Tabla 7

La contribución de las exportaciones al crecimiento económico, 1988-2004 (En porcentajes)

\begin{tabular}{lcccccc}
\hline Exportaciones & $\begin{array}{c}\text { Exportaciones } \\
\text { no petroleras / } \\
\text { exportaciones }\end{array}$ & $\begin{array}{c}\text { Cambios en } \\
\text { el volumen } \\
\text { petróleo / } \\
\text { exportaciones }\end{array}$ & $\begin{array}{c}\text { Cambio en } \\
\text { los precios } \\
\text { petroleros / } \\
\text { exportaciones }\end{array}$ & $\begin{array}{c}\text { Total } \\
\text { contribución } \\
\text { de factores / exportaciones }\end{array}$ & $\begin{array}{c}\text { Contribución } \\
\text { del cambio } \\
\text { en precios } \\
\text { petroleros / PIB }\end{array}$ \\
\hline Salinas & 30.14 & 115.47 & 0.03 & -15.49 & 100 & -4.67 \\
Zedillo & 115.35 & 93.97 & 1.61 & 4.42 & 100 & 5.09 \\
Fox & 64.21 & 81.38 & 8.06 & 10.56 & 100 & 6.78 \\
Total & 72.93 & 95.82 & 2.94 & 1.23 & 100 & 0.90 \\
\hline
\end{tabular}

Fuente: elaboración propia con datos de INEGI y Fox (2005). 
no residentes del país están financiando las crecientes importaciones de bienes y servicios. El financiamiento se registra en la cuenta financiera de la balanza de pagos que comprende la inversión extranjera directa, la inversión de cartera, el endeudamiento público o privado, u otras cuentas como la transferencia de recursos de los emigrantes mexicanos, que se incorporan antes de obtener el saldo de la cuenta corriente.

La tabla 8 considera la información de las transferencias brutas ${ }^{7}$ proporcionada por el INEGI y difiere ligeramente de la generada por el Banco de México quien detalla las remesas familiares por modalidad de transferencia, pero no representa la información previa a 1995. La primera columna se refiere a la contribución del ahorro externo (CAE) obtenida como el cociente de la variación absoluta del ahorro externo $\left(\left[\left(M_{T}-M_{T-\alpha}\right)-\left(D E_{T}-D E_{T-\alpha}\right)\right]\right)$ respecto a la variación absoluta del PIB, mientras que la segunda columna se refiere a la contribución de las trasferencias $(C T)$ estimada a partir de la aportación de las transferencias $\left(T_{T}-T_{T-\alpha}\right)$ al ahorro externo. La última columna evalúa la contribución de las transferencias (CTPIB) respecto a la variación absoluta del producto.

$$
C T=\left[\frac{\left(T_{T}-T_{T-\alpha}\right)}{\left(M_{T}-M_{T-\alpha}\right)-\left(D E_{T}-D E_{T-\alpha}\right)}\right] * 100
$$

$$
\text { CTPIB }=\left[\frac{\left(T_{T}-T_{T-\alpha}\right)}{\left(P I B_{T}-P I B_{T-\alpha}\right)}\right] * 100
$$

Bajo la lógica de la interpretación mencionada, durante el periodo Salinas el aho- rro externo fue importante para el financiamiento de la inversión y el crecimiento económico. En cambio, tuvo una menor importancia durante el periodo Zedillo y ninguna durante el de Fox, especialmente por la mayor expansión de las exportaciones de bienes y servicios respecto a lo que ocurrió con las importaciones. La contribución de las transferencias al ahorro externo también fue poco importante en los periodos de Salinas y Zedillo, mientras que ha sido más importante durante la administración de Fox. Al evaluar la aportación de las transferencias al crecimiento del PIB, se observa claramente que éstas fueron poco importantes en el gobierno Zedillo y ligeramente negativas durante el de Salinas, mientras que durante la administración de Fox explican 22.8\% de los incrementos -aunque limitados - de los ingresos y producto de la población.

\section{La contribución de la políticas fiscal y monetaria}

La determinación rigurosa de la contribución de la política fiscal es una cuestión compleja, en tanto que se debe evaluar el balance de lo que ocurre entre el gasto público - consumo y formación bruta de capital público-, que constituye una ayuda para el gasto privado, y los ingresos del sector público, que son equivalentes a una filtración de recursos. ${ }^{8}$ De manera más simple, la política fiscal activa puede identificarse con las declaraciones de política o la evidencia empírica, sobretodo cuando el gasto público crece en términos reales por encima del gasto privado. En este caso dividimos las cuentas del producto en gasto privado, gasto público y sector externo neto.

La tabla 9 presenta las tasas de crecimiento promedio del gasto privado y 
Cuadro 8

La contribución de las transferencias al ahorro externo y el crecimiento, 1988-2004 (En porcentajes)

\begin{tabular}{|c|c|c|c|}
\hline & \multirow[t]{2}{*}{$\begin{array}{l}\text { Ahorro } \\
\text { externo/PIB }\end{array}$} & \multicolumn{2}{|c|}{$\begin{array}{l}\text { Proporción de } \\
\text { transferencias externas }\end{array}$} \\
\hline & & $\begin{array}{l}\text { al ahorro } \\
\text { externo }\end{array}$ & al PIB \\
\hline \multicolumn{4}{|l|}{ Salinas } \\
\hline 1989 & 31.22 & -3.32 & -1.04 \\
\hline 1990 & 36.72 & 23.97 & 8.80 \\
\hline 1991 & 37.17 & -33.07 & -12.29 \\
\hline 1992 & 70.38 & 0.50 & 0.35 \\
\hline 1993 & -41.37 & 1.51 & -0.63 \\
\hline 1994 & 30.83 & 3.02 & 0.93 \\
\hline Total & 33.12 & -1.13 & -0.38 \\
\hline \multicolumn{4}{|l|}{ Zedillo } \\
\hline 1995 & 138.53 & -4.70 & -6.51 \\
\hline 1996 & 5.09 & 18.27 & 0.93 \\
\hline 1997 & 36.67 & 1.28 & 0.47 \\
\hline 1998 & 22.25 & 17.10 & 3.80 \\
\hline 1999 & 14.41 & -12.15 & -1.75 \\
\hline 2000 & 27.50 & -1.67 & -0.46 \\
\hline Total & -8.89 & -28.76 & 2.56 \\
\hline \multicolumn{4}{|l|}{ Fox } \\
\hline 2001 & $-2,232.68$ & 40.27 & -899.00 \\
\hline 2002 & 0.36 & $3,468.24$ & 12.43 \\
\hline 2003 & -45.64 & -94.13 & 42.96 \\
\hline 2004 & -4.26 & -256.21 & 10.93 \\
\hline Total & -1.70 & $-1,343.05$ & 22.81 \\
\hline 1988-2004 & 9.20 & 49.42 & 4.55 \\
\hline
\end{tabular}

Fuente: elaboración propia con datos de INEGI y Fox (2005).

público. En la mayor parte del periodo el gasto público real creció menos que el gasto privado. Sin embargo, en algunos años esa relación se revirtió como en 1993 durante el gobierno de Salinas y 1995 y 1999 durante el gobierno de Zedillo. Durante la administración Fox el gasto público es superior al gasto privado - 2002 y 2003 -

La política de mantener el balance fiscal en un déficit de alrededor de 1\% y el carácter procíclico de la política fiscal mexicana reciente, que aumenta o reduce
Tabla 9

La evolución del gasto privado y público, 1988-2004 (En millones de pesos constantes y porcentajes)

\begin{tabular}{|c|c|c|c|c|}
\hline & $\begin{array}{l}\text { Gasto } \\
\text { privado }\end{array}$ & $\begin{array}{l}\text { Variación } \\
(\%)\end{array}$ & $\begin{array}{l}\text { Gasto } \\
\text { público }\end{array}$ & $\begin{array}{l}\text { Variación } \\
(\%)\end{array}$ \\
\hline 1988 & $859,242.8$ & -- & $159,950.2$ & -- \\
\hline \multicolumn{5}{|l|}{ Salinas } \\
\hline 1989 & $911,134.0$ & 6.04 & $165,465.0$ & 3.45 \\
\hline 1990 & $977,428.5$ & 7.28 & $174,410.0$ & 5.41 \\
\hline 1991 & $1^{\prime} 036,394.7$ & 6.03 & $181,518.1$ & 4.08 \\
\hline 1992 & $1^{\prime} 109,040.1$ & 7.01 & $182,383.4$ & 0.48 \\
\hline 1993 & $1^{\prime} 119,686.5$ & 0.96 & $185,828.5$ & 1.89 \\
\hline 1994 & $1^{\prime} 170,680.1$ & 4.55 & $207,398.5$ & 11.61 \\
\hline Promedio & $1^{\prime} 054,060.7$ & 22.67 & $182,833.9$ & 14.31 \\
\hline \multicolumn{5}{|l|}{ Zedillo } \\
\hline 1995 & $999,910.9$ & -14.59 & $185,222.7$ & -10.69 \\
\hline 1996 & $1^{\prime} 074,183.2$ & 7.43 & $177,601.7$ & -4.11 \\
\hline 1997 & $1^{\prime} 186,076.1$ & 10.42 & $185,485.8$ & 4.44 \\
\hline 1998 & $1^{\prime} 270,904.2$ & 7.15 & $185,633.6$ & 0.08 \\
\hline 1999 & $1^{\prime} 322,217.4$ & 4.04 & $196,702.1$ & 5.96 \\
\hline 2000 & $1^{\prime} 434,293.2$ & 8.48 & $211,170.5$ & 7.36 \\
\hline Promedio & $1^{\prime} 214,597.5$ & 3.75 & $190,302.7$ & -8.24 \\
\hline \multicolumn{5}{|l|}{ Fox } \\
\hline 2001 & $1^{\prime} 450,925.5$ & 1.16 & $205,775.3$ & -2.55 \\
\hline 2002 & $1^{\prime} 455,149.2$ & 0.29 & $213,982.6$ & 3.99 \\
\hline 2003 & $1^{\prime} 461,468.8$ & 0.43 & $220,312.6$ & 2.96 \\
\hline 2004 & 1'527,539.6 & 4.52 & $220,799.2$ & 0.22 \\
\hline Promedio & $1^{\prime} 473,770.8$ & 2.75 & $215,217.4$ & 1.92 \\
\hline $\begin{array}{l}\text { Promedio, } \\
1989- \\
2004\end{array}$ & $1^{\prime 2} 219,189.5$ & 41.89 & $193,730.6$ & 21.12 \\
\hline
\end{tabular}

Fuente: elaboración propia con datos de Fox (2005).

el gasto público en la medida que los ingresos del sector público crecen o decrecen, parecen explicar la reducida contribución de esta política federal. No evaluamos los efectos de esta política en los ámbitos estatales o locales.

Agrupamos la información en gasto privado, gasto público y sector exportador neto - exportaciones menos importaciones-y aplicamos la fórmula descrita en la ecuación (2) para evaluar la contribución del gasto público al crecimiento económico. 
La tabla 10 muestra los resultados: éstos atribuyen una mayor contribución del gasto público al crecimiento de la producción agregada. Durante la administración de Salinas su aportación fue de $17.6 \%$, en la de Zedillo de 1.3\% y durante la de Fox de $9.2 \%$. Entonces, de acuerdo con esta metodología, los principales años de la contribución del gasto público real fueron 1994-1995 y 2001-2003.

Tabla 10

La contribución del gasto privado y público al crecimiento, 1988-2004 (En porcentajes)

\begin{tabular}{lrrr}
\hline & $\begin{array}{c}\text { Gasto } \\
\text { privado }\end{array}$ & $\begin{array}{c}\text { Gasto } \\
\text { público }\end{array}$ & $\begin{array}{c}\text { Sector } \\
\text { externo neto }\end{array}$ \\
\hline Salinas & & & \\
1989 & 118.61 & 12.61 & -31.22 \\
1990 & 120.46 & 16.25 & -36.72 \\
1991 & 122.41 & 14.76 & -37.17 \\
1992 & 168.37 & 2.01 & -70.38 \\
1993 & 44.30 & 14.33 & 41.37 \\
1994 & 91.94 & 38.89 & -30.83 \\
Total & 115.52 & 17.60 & -33.12 \\
Zedillo & & & \\
1995 & 211.11 & 27.41 & -138.53 \\
1996 & 117.10 & -12.02 & -5.09 \\
1997 & 127.67 & 9.00 & -36.67 \\
1998 & 122.03 & 0.21 & -22.25 \\
1999 & 94.11 & 20.30 & -14.41 \\
2000 & 112.92 & 14.58 & -27.50 \\
Total & 89.83 & 1.29 & 8.89 \\
Fox & & & \\
2001 & $-3,156.63$ & $1,023.95$ & $2,232.68$ \\
2002 & 34.10 & 66.26 & -0.36 \\
2003 & 27.16 & 27.20 & 45.64 \\
2004 & 95.04 & 0.70 & 4.26 \\
Total & 89.10 & 9.20 & 1.70 \\
Totales, & 100.09 & 9.11 & -9.20 \\
$1988-2004$ & & & \\
\hline
\end{tabular}

Fuente: elaboración propia con datos de Fox (2005).

Si la medición de la contribución de la política fiscal es controversial, la de la política monetaria es más compleja. La política monetaria sirve para controlar la inflación, reducir distorsiones y evitar otros factores nocivos para el crecimiento económico. Una inflación baja no garantiza el crecimiento del PIB, pero existe evidencia internacional de crecimiento positivo del PIB acompañado de una inflación moderada y excepcionalmente alta, como en Brasil durante los años setenta.

Desde 1987 el Banco de México ha señalado que el control de la inflación es una de sus prioridades, lo cual fue reafirmado en diciembre de 1993 (véase el artículo 2 de la Ley del Banco de México). ¿La búsqueda de inflación baja y estabilidad monetaria han ayudado al crecimiento económico? Ramos (1986) señala que teóricamente el objetivo de reducir la inflación no debe producir recesión y que, de hecho, ha habido programas que provocaron expansiones. No obstante, en la mayoría de los casos el impacto ha sido recesivo debido al desajuste de las expectativas, rigideces, desincronización en los contratos y falta de corrección de precios, así como la reducida credibilidad de las autoridades monetarias y la presencia de bienes y servicios imperfectos. En la misma dirección Ball (2000) señala que la desinflación implica costos, ubicándose entre la posición mayoritaria que señala que ésta reduce la producción y el empleo durante el periodo de transición hacia la inflación más baja y la posición pesimista que establece que la reducción en la producción no es sólo durante el periodo de transición sino que es indefinida debido a la "histéresis". 9

Existen algunas mediciones de los efectos de la inflación en la producción. Con tasas de inflación superiores a $40 \%$ la producción aumenta después de estabilizaciones exitosas. Barro (1996) señala que la inflación de $10 \%$ anual está asociada con una reducción de $0.3 \%$ a $0.4 \%$ del PIB anual. 
Así mismo, en los países de la OCDE, que tienen inflaciones menores a 20\%, el esfuerzo antiinflación redujo la producción entre $0 \%$ y $2 \%$, pero se elevó hasta $4 \%$ en un caso y en promedio el efecto fue de $0.82 \%$ (Yuravlivker y Partow, 2000). Ball (2000) señala que en Estados Unidos se podría perder entre $2 \%$ y $3 \%$ del PIB anual por un punto porcentual menos de inflación.

Este artículo no formula un modelo para evaluar la contribución del desempeño monetario y financiero al crecimiento económico. Por el contrario, emplea información estadística proveniente de las políticas monetarias y financieras nacionales y de otros países como Eu, eventos exógenos al control de las autoridades mexicanas y otros países, la interacción de las variables anteriores con la producción real, del sector externo, las finanzas públicas, entre otras como el mercado monetario y financiero mexicano. Lo anterior sirve para reiterar que la discusión de la exogeneidad y endogeneidad de la oferta monetaria y de otras variables financieras es debate vigente.

Los efectos del sector sobre el crecimiento son evaluados, en forma simplificada, a través de las variables de precio y cantidad como las tasas de interés y el acervo de crédito interno neto otorgado por el sistema bancario y financiero al sector privado — una "proxy" del aparato productivo- en porcentaje del PIB nominal; si éste es positivo entonces concluimos que contribuye al crecimiento del producto.

Durante el periodo analizado, el crédito neto al sector privado se comportó de la manera siguiente: en la administración de Salinas fue de $26.7 \%$ en promedio; Zedillo, 23.2\%; y Fox, 17.7\% (tabla 11). Esta tendencia indica que el sistema bancario contribuye cada vez menos al crecimiento de la producción. Si realizamos el análisis por años encontramos algunas tendencias interesantes. Durante todo el periodo de Salinas el crédito aumentó año con año, lo que se tradujo en una contribución positiva al crecimiento económico. Otra tendencia favorable sucedió en 2002 durante el gobierno de Fox, pero a partir de ahí la tendencia es descendente.

En el caso de las tasas de interés debemos prestar atención a dos elementos: el nivel real y el diferencial real entre las tasas locales y las internacionales, en este caso de Estados Unidos. Entre 1988 y 2004 las tasas de interés reales mexicanas se redujeron ${ }^{10}$ a pesar de los episodios alcistas de 1989, 1995 y 1998. Estas menores tasas contribuyeron a mayores niveles de gasto-demanda (consumo e inversión privada) y, por lo tanto, del producto ante la presencia de capacidad instalada ociosa. Sin embargo, la naturaleza contractiva de la política monetaria local se manifestó en que el diferencial entre las tasas reales del mercado monetario mexicano y el de EU fuera superior al diferencial entre las tasas reales de los bonos mexicanos respecto a los estadounidenses. Esto significó que se intentó imponer tasas en el mercado monetario que eran superiores al costo de obtener recursos de fuentes nacionales e internacionales. ${ }^{11}$

El comportamiento del crédito al sector privado y las tasas de interés se contradice en cuanto a su contribución al crecimiento económico. Las menores tasas de interés la promueven, ${ }^{12}$ pero la caída del crédito la restringe. Sin embargo, queda claro que la política monetaria tiende a imponer tasas de interés reales por encima del nivel de riesgo país y por arriba de las que resultan de las condiciones de los mercados financieros sin intervención de la autoridad monetaria. Debemos comentar que en años como 1996, pero especialmente 1997, debido al crecimiento de la captación y 
colocación de créditos, el diferencial de las tasas de interés se volvió negativo a pesar de la política monetaria restrictiva. No obstante, el análisis detallado de las tasas en cuanto a sus componentes exógenos y endógenos trasciende los alcances de este documento.

\section{La sobrevaluación de la moneda nacional}

La apreciación de la moneda nacional puede tener efectos reales sobre el crecimiento económico en la medida que eleva el poder de compra de la población que recibe ingresos en moneda nacional: tiene mayor poder de compra en bienes de consumo e inversión extranjeros y puede elevar su capacidad de endeudamiento en moneda extranjera. Estos elementos pueden promover un efecto acelerador en el consumo y la inversión privada la cual a su vez impulsa la expansión del producto. Cabe aclarar que este análisis no considera los efectos negativos de la apreciación de la moneda nacional sobre la asignación de recursos, la producción y la balanza de pagos en un horizonte de mediano y largo plazos.

Se propone un modelo simple de la cuenta corriente de la balanza de pagos, donde el poder de compra de la población se refleja en la demanda interna $(D I)$, pero si la moneda nacional se aprecia habría menores ingresos por las exportaciones de bienes y servicios $(D E)$. La magnitud de la apreciación cambiaria se mide con la diferencia entre el recíproco del tipo de cambio nominal $\left(\frac{1}{T C N}\right)$ y el tipo de cambio de paridad de poder de compra $\left(\frac{1}{T C P}\right)$. Para expresar estos resultados en moneda nacional y en porcentaje del producto, la fórmula anterior se multiplica por el tipo de cambio nominal y dividimos entre la diferencia del
Tabla 11

El desempeño de las principales variables financieras y monetarias, 1988-2004

\begin{tabular}{|c|c|c|c|c|}
\hline & \multicolumn{2}{|c|}{$\begin{array}{l}\text { Basado en } \\
\text { los precios al } \\
\text { consumidor }\end{array}$} & \multicolumn{2}{|c|}{$\begin{array}{l}\text { Crédito interno } \\
\text { sector privado/ } \\
\text { PIB }\end{array}$} \\
\hline & 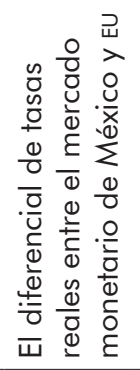 & 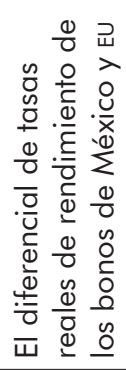 & 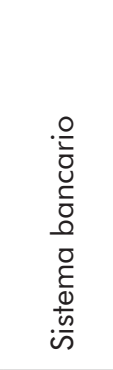 & 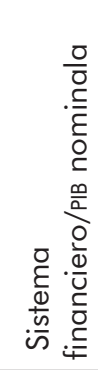 \\
\hline 1988 & -24.43 & -23.46 & 11.13 & 11.69 \\
\hline \multicolumn{5}{|l|}{ Salinas } \\
\hline 1989 & 18.60 & 17.62 & 15.58 & 16.35 \\
\hline 1990 & 5.98 & 4.48 & 17.47 & 18.34 \\
\hline 1991 & -0.61 & -4.65 & 20.96 & 22.00 \\
\hline 1992 & 2.37 & -0.38 & 28.07 & 29.47 \\
\hline 1993 & 6.80 & 4.61 & 31.76 & 33.34 \\
\hline 1994 & 7.40 & 5.12 & 38.74 & 40.67 \\
\hline Promedio & 6.76 & 4.47 & 25.43 & 26.70 \\
\hline \multicolumn{5}{|l|}{ Zedillo } \\
\hline 1995 & 16.26 & 7.33 & 29.27 & 30.72 \\
\hline 1996 & -2.92 & -4.32 & 18.80 & 19.74 \\
\hline 1997 & -1.98 & -3.35 & 25.26 & 26.52 \\
\hline 1998 & 5.78 & 4.47 & 22.54 & 23.74 \\
\hline 1999 & 3.63 & 1.63 & 19.22 & 20.41 \\
\hline 2000 & 4.05 & 2.86 & 17.18 & 18.33 \\
\hline Promedio & 4.14 & 1.44 & 22.04 & 23.24 \\
\hline \multicolumn{5}{|l|}{ Fox } \\
\hline 2001 & 5.04 & 3.98 & 14.63 & 16.06 \\
\hline 2002 & 3.02 & 2.05 & 16.92 & 18.82 \\
\hline 2003 & 3.21 & 2.75 & 15.98 & 18.11 \\
\hline 2004 & 3.66 & 3.32 & 15.59 & 17.71 \\
\hline Promedio & 3.73 & 3.03 & 15.78 & 17.67 \\
\hline $\begin{array}{l}\text { Promedio } \\
1989-2004\end{array}$ & 5.02 & 2.97 & 21.75 & 23.15 \\
\hline
\end{tabular}

a. Por falta de información, para 1988 y 1996 usamos la proporción del crédito al sector privado otorgado por el sector financiero con respecto al bancario en 1997.

Fuente: elaboración propia con datos de INEGI y FMI (2004). 
PIB del periodo a evaluar

$\left(P I B_{T}-P I B_{T-\alpha}\right)$ :

(12) $\left[\left(\frac{\left(D I_{T}-D I_{T-\alpha}\right)-\left(D E_{T}-D E_{T-\alpha}\right)}{P I B_{T}-P I B_{T-\alpha}}\right) *\right.$

$\left.\left(\frac{1}{T C N_{T}}-\frac{1}{T C P_{T}}\right) * T C N_{T}\right] * 100$

Debemos resaltar que este cálculo está sujeto a la discusión de la definición de tipo de cambio de paridad o "equilibrio". ${ }^{13}$ Sin embargo, el criterio de paridad de poder de compra tiene mucho sustento teórico y empírico. $\mathrm{Al}$ respecto, vamos a considerar el tipo de cambio de paridad como el producto del tipo de cambio nominal promedio compra-venta del año base, en este caso 1988, por el cociente de los índices de precios deflactores del PIB de México y EU con base homogénea en el año 2000.

La tabla 12 presenta el tipo de cambio promedio nominal, el tipo de cambio de paridad, la magnitud de la sobrevaluación del tipo de cambio y la contribución porcentual al crecimiento económico del fenómeno cambiario. Según la información del año base y los índices de precios, durante todo el periodo hay una clara apreciación del peso mexicano respecto al dólar americano, en promedio simple, equivalente a $30.6 \%$. Por periodos, la mayor apreciación se observa en la administración Fox, seguida por las de Salinas y Zedillo. En este último periodo llama la atención que aún en 1995 se mantuvo una ligera apreciación del peso mexicano.

En este sentido, en todo el periodo la apreciación de la moneda nacional elevó el poder de compra de la población en términos de bienes y servicios principalmente con origen en EU. Sólo se observan resultados negativos en 1995, porque la pérdida de ingresos de los exportadores de bienes y servicios fue superior al incremento del poder de compra del resto de la población. En general, este fenómeno cambiario es relevante y en promedio explica $22.7 \%$ del crecimiento económico; por sexenios, en la administración de Fox fue de $31.9 \%$, en la de Salinas de $23.8 \%$ y en la de Zedillo de $15.4 \%$.

Tabla 12

El tipo de cambio y su contribución al crecimiento económico, 1989-2004 (En pesos por dólar estadounidense y porcentajes)

\begin{tabular}{|c|c|c|c|c|}
\hline \multirow[b]{2}{*}{ Periodo } & \multicolumn{2}{|c|}{$\begin{array}{c}\text { En pesos por } \\
\text { dólar }\end{array}$} & \multicolumn{2}{|c|}{ En porcentajes } \\
\hline & 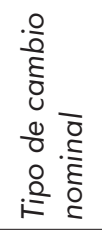 & 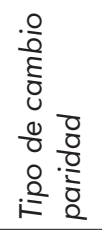 & 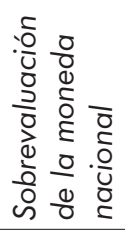 & 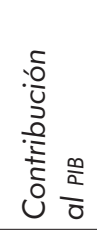 \\
\hline \multicolumn{5}{|l|}{ Salinas } \\
\hline 1989 & 2.46 & 2.77 & 11.14 & 9.86 \\
\hline 1990 & 2.81 & 3.42 & 17.83 & 15.45 \\
\hline 1991 & 3.02 & 4.07 & 25.79 & 25.98 \\
\hline 1992 & 3.09 & 4.55 & 32.00 & 34.34 \\
\hline 1993 & 3.12 & 4.87 & 36.06 & 28.51 \\
\hline 1994 & 3.38 & 5.17 & 34.69 & 28.72 \\
\hline Promedio & 2.98 & 4.14 & 28.06 & 23.81 \\
\hline \multicolumn{5}{|l|}{ Zedillo } \\
\hline 1995 & 6.42 & 6.98 & 8.04 & -0.39 \\
\hline 1996 & 7.60 & 8.95 & 15.12 & 9.50 \\
\hline 1997 & 7.92 & 10.37 & 23.63 & 20.16 \\
\hline 1998 & 9.14 & 11.83 & 22.78 & 18.06 \\
\hline 1999 & 9.56 & 13.43 & 28.80 & 19.48 \\
\hline 2000 & 9.46 & 14.73 & 35.83 & 25.54 \\
\hline Promedio & 8.35 & 11.05 & 24.44 & 15.39 \\
\hline \multicolumn{5}{|l|}{ Fox } \\
\hline 2001 & 9.34 & 15.23 & 38.66 & 54.58 \\
\hline 2002 & 9.66 & 16.01 & 39.69 & 31.36 \\
\hline 2003 & 10.79 & 17.02 & 36.61 & 22.29 \\
\hline 2004 & 11.29 & 17.63 & 35.97 & 19.28 \\
\hline Promedio & 9.88 & 15.39 & 35.76 & 31.88 \\
\hline $\begin{array}{l}\text { Promedio 1989- } \\
2004\end{array}$ & 6.81 & 9.81 & 30.56 & 22.67 \\
\hline
\end{tabular}

Fuente: elaboración propia con datos del Bureau of Economic Analysis, INEGI y Fox (2005). 
El gobierno debería evitar la apreciación del tipo de cambio porque ésta promueve una inadecuada asignación de recursos que alienta las importaciones de bienes y servicios. La situación es particularmente delicada cuando nos referimos a las importaciones sustitutas de bienes de consumo y en menor medida a las complementarias. La importación de bienes de capital no resulta ser tan negativa, ya que generalmente contribuye a elevar la capacidad productiva de la economía, a menos que eso signifique dejar de comprar bienes de capital de origen nacional.

\section{Conclusión}

El crecimiento del PIB en nuestro país muestra una tendencia decreciente desde la administración de Salinas. En la presente administración el producto per cápita ha crecido ligeramente por encima de $0 \%$.

El presente artículo no hace una comparación internacional, pero no es difícil ver que los resultados de México son mediocres si los comparamos con el desempeño de los principales países asiáticos y algunos latinoamericanos. Se obtuvieron resultados poco satisfactorios a pesar de que cada administración introdujo reformas económicas para modernizar y articular mejor a la economía mexicana con los mercados internacionales.

También destaca la profundización de los viejos desajustes estructurales como la expansión del sector terciario de la economía, que durante la administración de Fox aporta $96.3 \%$ del crecimiento económico esperábamos que la integración de México a la economía estadounidense impulsara al sector secundario o de transformación, situación que no sucedió-. Además, dentro del sector terciario destaca la participación del subsector financiero, seguros y bienes inmuebles, seguido por el de transporte, almacenamiento y comunicaciones. Esa participación contradice a la aportación del primer subsector al PIB a través del crédito interno al sector privado otorgado por el sistema bancario y financiero, ya que éste ha disminuido recientemente.

Llama la atención que durante la administración Fox el crecimiento se ha basado en la demanda a través de la expansión del consumo, mientras que la aportación de la formación bruta de capital privado y la variación de existencias es negativa. La administración "del cambio" no ha logrado, hasta el momento, la movilización de la inversión privada. La política de reducción de las tasas del impuesto a la renta, heredadas de la administración Zedillo, no parece tener efecto alguno en la promoción de la inversión productiva.

Mientras las condiciones del entorno internacional no fueron tan favorables para Salinas, sí lo fueron para Zedillo y Fox quienes gozaron de buenos precios internacionales del petróleo, lo cual implica que en la actual administración el reducido crecimiento económico debe atribuirse más a razones de origen interno que externo. Sin embargo, la desaceleración productiva de los EU a partir del primer trimestre de 2001 y los acontecimientos de septiembre del mismo año afectaron en forma evidente a nuestro desempeño económico.

No todo es malo o mediocre, ya que las exportaciones de la industria maquiladora de exportación explican $40.5 \%$ del crecimiento económico observado durante 1988-2004. Sin embargo, no debemos olvidar que $76.2 \%$ de esas exportaciones son filtraciones hacia el exterior en la modalidad de importaciones de bienes y servicios, pero eso es tema de otro trabajo. Obviamente, con relación a las otras administraciones la mayor aportación de 
la actividad maquiladora se dio durante la administración Zedillo, quien cosechó los resultados de la firma del TLCAN.

Nuestra evaluación señala que la contribución de la política fiscal al crecimiento económico fue baja y de la política monetaria ha sido negativa desde el gobierno zedillista. Por la naturaleza procíclica de la política fiscal, salvo en años específicos, el gasto público real creció por debajo del gasto privado real. Contrario a lo que se pudiera pensar, las mayores aportaciones de la política fiscal se dieron durante el gobierno Salinas, seguido por Fox y Zedillo, donde a pesar de la crisis, la contribución de la formación bruta de capital del sector público fue negativa.

Fue difícil conocer la contribución de la política monetaria. Obviamente, la lucha contra la inflación tiene costos sobre el crecimiento económico. La reducción de las tasas de interés reales favorece al incremento de la demanda y la producción, mientras que la reducción del crédito interno neto al sector privado desde el gobierno de Zedillo afectó negativamente al crecimiento del PIB.

Sólo en la administración de Salinas las políticas monetarias de lucha contra la inflación parecen haber logrado efectos positivos en los niveles de crédito interno al sector privado. Por el contrario, a pesar del sesgo de las políticas de la administración Fox en favor de lo financiero, la participación del crédito interno ha decrecido. El sector financiero se vanagloria de su crecimiento, pero su aportación en términos del crédito interno al sector privado es ahora un poco más elevada que en 1989.

A pesar de su bajo crecimiento, el gobierno Fox ha sido muy afortunado por tres factores ajenos a las decisiones de política económica: el incremento de los precios internacionales del petróleo crudo y de la mezcla mexicana de exportación, los ingresos por remesas de los mexicanos residentes en el exterior y la apreciación del peso. En los tres casos se trata de choques de ingresos que promueven el incremento de la demanda y la producción. Los tres factores, en nuestra estimación preliminar, explican $61.5 \%$ del crecimiento del PIB en la administración de Fox, 23\% en la de Zedillo y $18.8 \%$ en la de Salinas. En la actual administración el aumento de los precios del petróleo exportado explica $6.8 \%$ del crecimiento económico, mientras que en las administraciones de Zedillo y Salinas fue de $5.1 \%$ y $-4.7 \%$, respectivamente. Es justo decir que en el actual gobierno el incremento de los volúmenes exportados explica $8.1 \%$ del crecimiento de las exportaciones frente a $1.6 \%$ en la administración de Zedillo y $0 \%$ en la de Salinas. Por último, las transferencias de los mexicanos en el exterior explican 22.8\% del crecimiento del PIB durante el periodo de Fox, $2.6 \%$ en el de Zedillo y $0.7 \%$ en el de Salinas.

El aumento acelerado de las importa- 
ciones sustitutivas durante este gobierno se explica por la apreciación de la moneda y el consumo privado, que contribuyeron negativamente al producto con $37.7 \%$, $22.3 \%$ en la administración de Zedillo y $11.7 \%$ en la de Salinas. Sin embargo, este crecimiento basado en el consumo, contrarrestado por las importaciones de bienes de consumo, proporcionaría un excelente espacio para la acción durante el próximo gobierno, conjuntamente con la transformación de la política fiscal en una herramienta contra cíclica. my

\section{Notas}

1 Lamentablemente, la información de las cuentas nacionales con base 1980 del INEGI no es compatible con la base más reciente.

2 De acuerdo con lo señalado por Felipe y McCombie (2005).

3 Se explican principalmente por el nivel y la estructura del PIB, el grado de apertura de la economía y el tipo de cambio real.

4 La fórmula utilizada es similar a la presentada en la ecuación (1).

5 A menos que se trate de bienes que no puedan producirse localmente. Sin embargo, los bienes de consumo son más difícil de sustituirlos por productos de origen nacional.

6 No pretendemos discutir la causalidad del ahorro a la inversión o viceversa.

7 El INEGI las define como operaciones sin contrapartida en bienes, servicios u de operaciones financieras, explicadas principalmente por las remesas de los emigrantes menos las remesas de los inmigrantes y otras operaciones.

8 Los mayores ingresos del sector público debidos a los mayores precios de los hidrocarburos no reducen los ingresos del sector privado.

9 Concepto acuñado por Blanchard y Summers (1996) para describir los efectos permanentes de la desinflación, mediante la cual la elevada tasa natural de desempleo permanece en su nuevo nivel luego del proceso de corrección de los precios absolutos. Las explicaciones de este fenómeno son diversas: la erosión de las destrezas de los trabajadores, las modificaciones en la oferta de trabajo y la menor probabilidad de que los empleadores contraten a trabajadores desocupados.

10 No se debe olvidar la existencia de tasas de interés reales negativas de los fondos federales entre 2003 y 2004 y que disminuyeron entre 1999-2001.

11 Una medida del riesgo país es el diferencial de las tasas de rendimiento real de los bonos mexicanos menos los norteamericanos.

12 La reducción en las tasas de interés reales es menor que la disminución en los niveles de crédito interno al sector privado, por lo que el efecto contractivo parece dominar.

13 Se elude el análisis de la contribución positiva o negativa de las cuentas financieras de la balanza de pagos y si el sector externo se encuentra en equilibrio.

\section{Referencias bibliográficas}

Ball, L. (1993), "What determines the sacrifice ratio", NBER Working Paper, núm. 4306, Cambridge, MA. (2000), "Los costos de la desinflación", en Revista Ensayos sobre Política Económica (Banco de la República de Colombia), núm. 37, pp. 63-76.

Cárdenas, E. (1994), La hacienda pública y la política económica, 1929-1958, Fondo de Cultura Económica y El Colegio de México, México, DF, $188 \mathrm{pp}$.

- (1996), La política económica en México, 19501994, Fondo de Cultura Económica y El Colegio de México, México, DF, 256 pp.

Díaz de León, J. (2005), "México: aplicación del multiplicador de las remesas", en Economía Informa (UNAM), núm. 336, pp. 124-131.

Felipe, J. y J. McCombie (2005), "La función de producción agregada en retrospectiva", en Investigación Económica (UNAM), vol. 64, núm. 253, pp.43-88.

FMI (2004), International Financial Statistics Yearbook, Washington, DC.

Fox, V. (2005), Quinto Informe de Gobierno. Anexo estadístico, Presidencia de la República, México, DF, 2005.

Marshall, J. (1986), "Modelos y políticas de crecimiento", en R. Cortázar (ed.), Políticas macroeconómicas. Una perspectiva latinoamericana, Cieplan, Santiago de Chile, pp. 325-363.

Ramos, J. (1986), "Políticas de estabilización", en R. Cortázar (ed.), Políticas macroeconómicas. Una perspectiva latinoamericana, Cieplan, Santiago de Chile, pp. 17-59.

Yuravlivker, D. y Z. Partow (2000), "Inflación intermedia en los países andinos del norte y los costos de la desinflación: la experiencia internacional", en Revista Ensayos sobre Política Económica (Banco de la República de Colombia), núm. 37, pp. 243-286. 